\title{
France wires up to treat obsessive disorder
}

\section{Sally Goodman, Paris}

France's ethics commission has given the goahead for clinical trials of neurosurgery on patients with obsessive-compulsive disorder (OCD), a debilitating psychiatric condition.

The ruling by the National Consultative Committee on Ethics will allow researchers to conduct carefully controlled trials using neurostimulation - a technique that involves stimulating a targeted area of the brain using implanted electrodes connected to a battery.

Neurosurgery was virtually abandoned as a treatment of psychiatric illness throughout the world after public revulsion over the abuse of lobotomies half a century ago. However, progress in neuroscience has led to renewed interest in such interventions, raising some thorny ethical questions.

Neurostimulation has the advantage of being reversible and, because the stimulator can be switched on and off and the electrodes removed, blind control trials can be carried out on consenting patients. OCD is a leading contender for such trials because it is well understood and therefore easy to target in the brain. What's more, its sufferers, unlike most other psychiatric patients, can often give informed consent to surgery. In both the United States and Europe, ethics committees decide whether the patients can give consent on a case-by-case basis.
Last October, Alim-Louis Benabid, a neurosurgeon at the Joseph Fourier University in Grenoble, asked the French commission to consider the ethics of using neurostimulation on OCD patients. The technique has been used to treat movement disorder in Parkinson's disease, but it has been tried on only about 20 OCD patients in Belgium, Sweden and the United States in the past five years.

Experimental surgical techniques are not constrained by France's bioethics laws, but Benabid says that he wanted an opinion from the ethics commission before starting clinical trials. The commission decided in favour of using neurostimulation, but said that strict experimental conditions should apply, including the setting up of a committee to oversee the choice of patients, their consent, and the evaluation of results.

The commission added that the experiment should involve only the most severe cases of OCD. Sufferers perform rituals, such as washing their hands, hundreds of times a day. Around $1-2 \%$ of people are thought to be affected, with some $10 \%$ of sufferers showing severely debilitating symptoms.

Bart Nuttin, a neurosurgeon from the Catholic University of Leuven in Belgium, told the commission that he was working with others to draft a scientific and ethical framework for using the technique before it

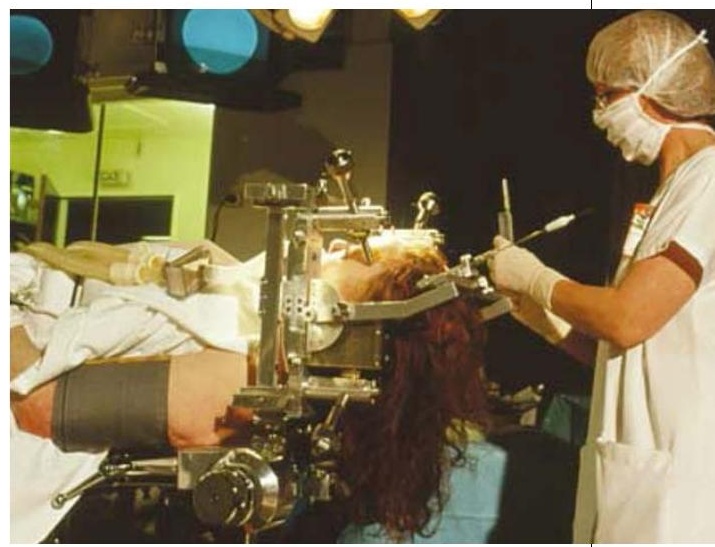

Tried and tested: neurostimulation has been used to treat patients with Parkinson's disease.

becomes widely established.

"We were very concerned about this technique falling into the wrong hands," Nuttin says, adding that the journal Neurosurgery has accepted the group's proposed guidelines for publication later this year.

However, progress in functional brain imaging is also tempting researchers to try to use neurostimulation to treat other psychiatric illnesses, such as depression, where such issues as the identification of the organic basis of the disorder and informed consent are even more complex.

\section{Statistical error leaves pollution data up in the air}

\section{Jonathan Knight, San Francisco}

An off-the-shelf statistics package has tripped up pollution researchers in North America and Europe who are studying the effects of airborne soot on human health.

A default setting that produced erroneous results went unchecked for years, despite significant statistical expertise in all of the groups. "It was already such standard software when we started using it, I didn't

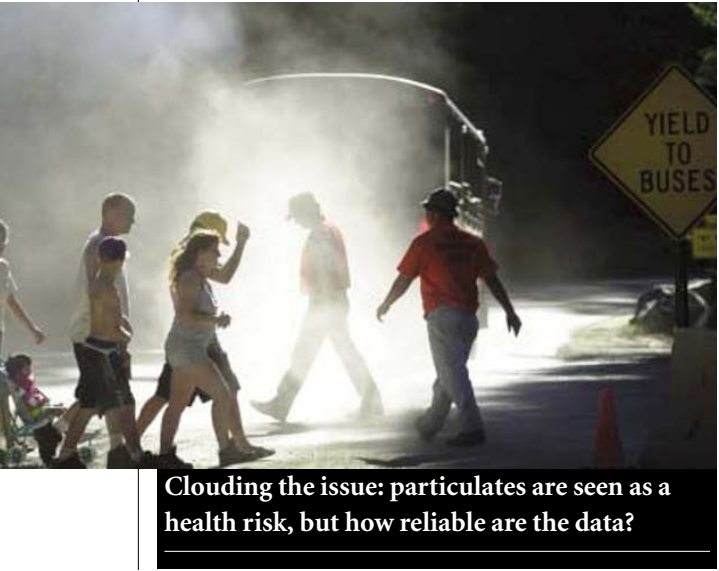

even question it," says Francesca Dominici, a public-health researcher at Johns Hopkins University in Baltimore, Maryland.

On 4 June, Dominici posted revised figures on her website after discovering that the error had doubled her group's estimate of the risk to health posed by particulates in the air. Two other groups that used the same tool, one in Canada and one in Greece, are now redoing their calculations.

The groups were looking for correlations between death rates and particulates in the air, which come mainly from diesel engines and power plants. Their data on air quality, hospitalizations and deaths from dozens of cities cover a seven-year period up to 1994 .

Death rates vary throughout the year because of such factors as changes in temperature and disease outbreaks. To tease out the effects of particulates, the groups used a statistics program known as S-Plus.

S-Plus searches for correlations using an iterative process in which confounding effects are gradually peeled away. The default parameter in question determined how many times the procedure would iterate before stopping to produce a final result.
"For most applications the value is perfectly fine," says David Smith, product manager of Seattle-based Insightful, which sells S-Plus. Smith says that the Hopkins case was exceptional, but that users should always check whether changing the parameter affects the outcome, and adjust it if necessary. Smith says that Insightful will tighten the default value of the parameter slowing the programme slightly - on future releases of S-Plus.

Richard Burnett, a statistician with Health Canada in Ottawa, which is conducting a similar study, says that his group will probably revise its estimates of the impact of airborne soot on mortality downwards by $20-50 \%$. The findings of a study run by a group at the University of Athens may also have to be adjusted, he says.

The health risk posed by particulates is a source of fierce environmental controversy in the United States, and the Bush administration is considering rules to restrict emissions. Opponents of tighter rules are likely to seize on the revisions as evidence that the research linking soot in the air to harmful effects on health is not to be trusted. 